\title{
How effective is elagolix treatment in women with fibroids and HMB?
}

\section{Elagolix with hormonal add-back therapy (estradiol/ norethindrone) was effective: $\mathbf{8 7 . 9 \%}$ of participants who received combination therapy met both primary endpoints:}

1) percentage of women with less than $80 \mathrm{~mL}$ menstrual blood loss during the final month, and 2) a $50 \%$ or greater reduction in menstrual blood loss from baseline to final month. Treatment was for up to 12 months as an extension of 1 of 2 randomized, double-blinded, placebo-controlled studies using elagolix.

Simon JA, Al-Hendy A, Archer DF, et al. Elagolix treatment for up to 12 months in women with heavy menstrual bleeding and uterine leiomyomas. Obstet Gynecol. 2020;135:1313-1326.

\section{EXPERT COMMENTARY}

Andrea S. Lukes, MD, MHSc, is Founder, Carolina Women's Research and Wellness Center, and Chief Medical Officer, Health Decisions Inc., Durham, North Carolina.

\section{U} terine fibroids are common (occurring in up to $80 \%$ of reproductive-age women), ${ }^{1,2}$ and often associated with heavy menstrual bleeding (HMB). There are surgical and medical options, but typically medical options are used for short periods of time. Elagolix with hormonal add-back therapy was recently approved (May 29, 2020) by the US Food and Drug Administration (FDA) for treatment of HMB in women with uterine fibroids for up to 24 months.

Dr. Lukes reports being the Principal Investigator for Abbvie, Myovant, and Obseva; a consultant for Abbvie, Myovant, and Antev; a speaker for Abbvie; a member of the Liberty Steering Committee for Myovant; and an investigator for Abbvie, Myovant, Obseva, Merck, Bayer, Sequoia, Ferring, and Sebela. 
Elagolix is an oral, nonpeptide gonadotropin-releasing hormone antagonist that results in a dose-dependent reduction of gonadotropins and ovarian sex hormones. There are now 2 approved products containing elagolix, with different indications:

- Orilissa. Elagolix was approved in 2018 by the FDA for moderate to severe pain associated with endometriosis. For that indication there are 2 dose options of elagolix (150 mg for up to 2 years and $200 \mathrm{mg}$ for up to 6 months) and there is no hormonal add-back therapy.

- Oriahnn. Elagolix and hormonal add-back therapy was approved in 2020 for HMB associated with uterine fibroids for up to 24 months. The total daily dose of elagolix is $600 \mathrm{mg}$ (elagolix $300 \mathrm{mg}$ in the morning with estradiol $1 \mathrm{mg} /$ norethindrone acetate $0.5 \mathrm{mg}$ and then in the evening elagolix 300 mg and no hormonal add-back).

This new class of drug, GnRH antagonist, is an important one for women's health, and emerging science will continue to expand its potential uses, such as in reproductive health, as well as long-term efficacy and safety. The difference in daily dose of elagolix for endometriosis (150 mg for 24 months) compared with HMB associated with fibroids (600 mg for 24 months) is why the hormonal add-back therapy is important and allows for protection of bone density.

This is an important manuscript because it highlights a medical option for women with HMB associated with fibroids, which can be used for a long period of time. Further, the improvement in bleeding is both impressive and maintained in the extension study. Approximately $90 \%$ of women show improvement in their menstrual bleeding associated with fibroids.

The question of what to do after 24 months of therapy with elagolix and hormonal add-back therapy is an important one, but providers should recognize that the limiting factor with this elagolix and hormonal addback therapy is bone mineral density (BMD). We will only learn more and more moving forward if this is a clinically meaningful reason for stopping treatment at 24 months. The
FDA takes a strict view of safety, and providers must weigh this with the benefit of therapy.

One other highlight between the 2 approved medications is that Orilissa does not have a black box warning, given that there is no hormonal add-back therapy. Oriahnn does have a warning, regarding thromboembolic disorders and vascular events:

- Estrogen and progestin combinations, including Oriahnn, increase the risk of thrombotic or thromboembolic disorders, especially in women at increased risk for these events.

- Oriahnn is contraindicated in women with current or a history of thrombotic or thromboembolic disorders and in women at increased risk for these events, including women over 35 years of age who smoke or women with uncontrolled hypertension.

\section{Details about the study}

The study by Simon et al is an extension study (UF-EXTEND), in that women could participate if they had completed 1 of the 2 pivotal studies on elagolix. The pivotal studies (Elaris UF1 and UF2) were both randomized, double-blinded, placebocontrolled studies with up to 6 months of therapy; for UF-EXTEND, however, participants were randomly assigned to either combined elagolix and hormone replacement therapy or elagolix alone for an additional 6 months of therapy. Although it was known that all participants would receive elagolix in UF-EXTEND, those who received hormonal add-back therapy were blinded. All women were then followed up for an additional 12 months after treatment ended.

The efficacy of elagolix was measured by the objective alkaline hematin method for menstrual blood loss with the a priori coprimary endpoints. The elagolix and hormonal add-back therapy group showed objective improvement in menstrual blood loss at 12 months in $87.9 \%$ of women in the extension study (89.4\% in the elagolix alone group). This compares with $72.2 \%$ improvement at 6 months of treatment in the UF1 and UF2 studies for those taking elagolix and hormonal add-back therapy. These findings
FAST TRACK

For those women who experienced a decrease in bone density, the reduction was less than $5 \%$ for the lumbar spine 


\section{WHAT THIS EVIDENCE MEANS FOR PRACTICE}

Elagolix and hormonal add-back therapy offer a long-term medical option for women with $\mathrm{HMB}$ associated with fibroids that is both effective and safe. to 12 months of treatment at month 6 in the extension study. After stopping elagolix with add-back therapy, at 6 months the elagolix with add-back therapy had a Z-score of $-0.6 \%$ (95\% CI, -1.1 to -0.1 ). This shows a trend toward baseline, or a recovery within a short time from stopping medication.

\section{Study strengths and limitations}

Strengths of this study include its overall design; efficacy endpoints, which were all established a priori; the fact that measurement of menstrual blood loss was done with the objective alkaline hematin method; and the statistical analysis, which is thorough and well presented. This extension study allowed further evaluation of efficacy and safety for elagolix. Although the authors point out that there may be some selection bias in an extension study, the fact that so many women elected to continue into the extended study is a positive reflection of the treatment.

As providers learn of new therapies for management of HMB associated with fibroids, it is important to consider who will benefit the most. In my opinion, any woman with heavy periods associated with fibroids could be a candidate for elagolix with addback therapy. This treatment is highly effective, well tolerated, and safe. My approach to management includes educating a woman on all potential therapies and this new option of elagolix and add-back therapy is an important one. The decision for an individual woman on how to manage heavy periods associated with fibroids should consider her contraceptive needs, medical issues, and the risk and benefit of individual therapies.

\footnotetext{
References

1. Stewart EA, Nicholson WK, Bradley L, et al. The burden of uterine fibroids for African-American women: results of a national survey. J Women's Health. 2013;22:807-816.

2. Baird DD, Dunson DB, Hill MC, et al. High cumulative incidence of uterine leiomyoma in black and white women: ultrasound evidence. Am J Obstet Gynecol. 2003;188:100-107.
} 